\title{
Overexpression of the Shb SH2 Domain-Protein in Insulin-Producing Cells Leads to Altered Signaling Through the IRS-1 and IRS-2 Proteins
}

\author{
Nils Welsh, Natalia Makeeva, and Michael Welsh \\ Department of Medical Cell Biology, Uppsala University, Uppsala, Sweden \\ Contributed by D. Steiner. Accepted September 9, 2002
}

\begin{abstract}
Background: Overexpression of the Src homology 2 domain protein Shb in $\beta$-cells of transgenic mice has been shown to promote an increased $\beta$-cell mass. To investigate the mechanisms by which Shb controls the $\beta$-cell mass, we have presently studied the effects of Shb overexpression on the IRS-1-induced signaling pathway in mouse islet $\beta$-cells and in insulin-producing RINm5F cells and correlated these effects to growth and death patterns.

Materials and Methods: Shb overexpression was achieved in RINm5F cells by selection of stable clones or by FACS purification of transiently transfected cells. For Shb overexpression in primary mouse islet cells, a Shb-transgene mouse was used. Cell proliferation and death rates were determined using flow cytometry. Serum-, insulin-, and IGF-1stimulated signaling events were studied by immunoblot, immunoprecipitation, and in vitro kinase procedures.
\end{abstract}

Results: Transient Shb overexpression in RINm5F cells resulted in increased proliferation. Both Shb-overexpressing RINm5F cells and islet cells from transgenic mice (islet $\mathrm{Shb}$ ) exhibited increased basal tyrosine phosphorylation of IRS-1. Shb overexpression resulted also in the assembly and activation of a multiunit complex consisting of at least Shb, IRS-1, IRS-2, FAK, and PI3K. Consequently, the phosphorylation of Akt was enhanced under basal conditions in Shb overexpressing cells. Finally, Shb overexpression did not affect insulin-induced phosphorylation of the PI3K-antagonist PTEN.

Conclusion: It is concluded that the Shb-induced alterations in the IRS-1/PI3K/Akt pathway may be relevant to the understanding of growth and death patterns of insulin-producing cells.

\section{Introduction}

The development of diabetes mellitus depends on the balance between $\beta$-cell proliferation and death (1). Recent investigations have implicated insulin or insulin-like growth factor-1-dependent (IGF) signaling via the insulin receptor substrates (IRS1, IRS-2) of paramount importance for $\beta$-cell proliferation, survival, and insulin gene expression (2-5). Whereas inactivation of the IRS-1 gene increases the $\beta$-cell mass, inactivation of the IRS-2 gene reduces the number of $\beta$-cells (2). The increased $\beta$-cell mass after IRS- 1 gene inactivation could be the consequence of a compensatory response to peripheral insulin resistance, because $\beta$-cell-specific gene inactivation of the insulin receptor gene caused a modest reduction of the pancreatic $\beta$-cell mass at 4 months of age (6). The reduced $\beta$-cell mass in the IRS-2 knockout mice implies a significant role for this signaling protein in $\beta$-cell replication or survival. Inactivation of IGF-1 receptor gene further accentuated the decrease in the $\beta$-cell mass when such mice were crossed with IRS-2-deficient mice (4), implying signaling through the IGF-1 receptor as relevant for the effects of IRS-2 on $\beta$-cell replication and/or

Address correspondence and reprint requests to: Dr Nils Welsh, Department of Medical Cell Biology, Box 571, Biomedicum, S-75123, Uppsala, Sweden. Phone: 46-18-4714212; fax: 46-18-556401; e-mail: Nils.Welsh@medcellbiol.uu.se. survival. Indeed, increased $\beta$-cell apoptosis was noted in IGF-1 receptor/IRS-2 knockout mice (4).

It is well established that IRS-1 and IRS-2 signal in part by activating the ERK and PI3K/Akt pathway. PI3K activation leads to the phosphorylation of PtdIns, PtdIns-4-P, PtdIns4,5- $\mathrm{P}_{2}$ to generate PtdIns-3-P, PtdIns-3,4- $\mathrm{P}_{2}$, or PtdIns-3, 4, 5- $\mathrm{P}_{3}$ (7). PtdIns-3,4- $\mathrm{P}_{2}$ recruits and activates Akt (8), whereas PtdIns-3,4,5- $\mathrm{P}_{3}$ recruits and activates the Akt-phosphorylating kinase PDK 1 (9). Akt, in turn, when phosphorylated by PDK 1 in position Thr308 and by an unknown kinase in position Ser473 (10), promotes cell survival by phosphorylating BAD (11), caspase-9 (12), the forkhead transcription factor FKHRL1 (13), and other substrates. The discovery of the tumor suppressor PTEN adds further complexity to the regulation of this pathway. This is a phosphatase, which dephosphorylates the third position of PtdIns-phosphates, thereby antagonizing the activity of PI3K $(14,15)$.

Shb is a Src homology 2 (SH2)-domain adapter protein with proline-rich motifs in its $\mathrm{N}$-terminus, a central phosphotyrosine binding (PTB) domain and a C-terminal SH2 domain $(16,17)$. Shb has been found to interact with the PDGF receptors, FGF receptor- 1 , and T-cell receptor $\zeta$-chain via its $\mathrm{SH} 2$ domain $(17,18)$, to LAT via its PTB domain $(17,19)$ and to Src, Eps8, p85 PI-3 kinase, Grb2, and PLC $\gamma$ via its proline-rich motifs (17-19). When Shb was 
overexpressed in NIH3T3 cells, increased apoptosis upon serum withdrawal was observed (20).

To address a role for Shb in $\beta$-cell function, a transgenic mouse overexpressing shb under the control of the rat insulin promoter was generated (21). These mice exhibit slightly improved glucose tolerance, an increased $\beta$-cell mass at birth that persisted up to the age of 6 months, increased $\beta$-cell apoptosis during tissue culture at a low serum concentration, and increased susceptibility to multiple injections of streptozotocin in vivo (21). The present study was conducted to elucidate the signaling pathways responsible for the effects of Shb on growth and survival of $\beta$-cells, taking particular notice of the IRS-1/IRS-2 signaling system.

\section{Materials and Methods}

\section{RIN-Shb Cells and Shb Islets}

A RINm5F cell clone, which continuously overexpresses Shb (RIN-Shb), and a clone transfected with the neomycin resistance gene only (RIN-Neo), were generated in a previous study (20). Growing RINm5F cells (passage number $>120$ ), RIN-Shb, and RIN-Neo cells were trypsinized every 3-5 days and subcultured in RPMI 1640 supplemented with $10 \%$ fetal calf serum (FCS), $2 \mathrm{mM}$ L-glutamine, benzylpenicillin $(100 \mathrm{U} / \mathrm{ml})$, and streptomycin $(0.1 \mathrm{mg} / \mathrm{ml})$ at $37{ }^{\circ} \mathrm{C}$ in humidified air with $5 \%$ $\mathrm{CO}_{2}$. The Shb transgene has been described (21). For experimentation, Shb transgenic mice of both sexes at approximately 3 months of age were used. Islets from Shb-transgenic and CBA control mice were isolated as described (22). Islets were precultured for 2-5 days under the conditions described above before experimentation.

\section{FACS Sorting of Shb- + Green Fluorescent Protein- Transfected Cells}

RINm5F cells were transfected with the ShbpcDNAl vector and pd4EGFP-Nl (5:1, w/w) (Clontech, Palo Alto, CA, USA) using Lipofectamine (Gibco BRL, Gaithersburg, MD, USA) as described (23). Control cells were transfected with pd4EGFP-N1 and mock DNA only. The following day, green cells $(4-10 \%)$ were sorted using the FACSCalibur flow cytometer (Becton Dickinson, San Jose, CA, USA). Sorted and nonsorted cells were concentrated by centrifugation, plated in wells, and allowed to grow for another 1-2 days before further experimentation as described below.

\section{Quantification of Cell Proliferation and Apoptosis by Flow Cytometry}

Two thousand green fluorescent protein (GFP)-RIN or GFP + Shb-RIN cells were plated at day 0 (day of cell sorting) and cultured as described. On days 1,2 , and 3 the cells were stained for 10 min with
$10 \mu \mathrm{g} / \mathrm{ml}$ of propidium iodide (PI) and washed with phosphate-buffered saline (PBS). The cells were then detached by trypsination and resuspended in $400 \mu \mathrm{l}$ of culture medium. The cells were analyzed in a Becton Dickinson FACSCalibur flow cytometer with respect to their forward scatter and $\mathrm{FL}_{3}$-fluorescence. Cells with low, intermediate, or high FL3-fluorescence are viable, apoptotic, or necrotic, respectively (24). Data were analyzed using the CellQuest software (Becton Dickinson).

\section{Immunoprecipitation of IRS-1/IRS-2}

RIN-Shb- and RIN-Neo cells $\left(10 \times 10^{6}\right)$ or GFPRIN and GFP + Shb-RIN cells $\left(2-3 \times 10^{5}\right)$ were maintained in RPMI 1640 without serum for 60 min. Insulin $(0.1$ or $10.0 \mu \mathrm{g} / \mathrm{ml})$, FCS $(10 \%)$, or IGF-1 $(100 \mathrm{ng} / \mathrm{ml})$ was added to some groups of cells. After $10 \mathrm{~min}$, cells were washed in cold PBS containing $100 \mu \mathrm{M}$ of $\mathrm{Na}_{3} \mathrm{VO}_{4}$ and lysed in 100 $\mathrm{mM}$ Tris, pH 8.0, $150 \mathrm{mM} \mathrm{NaCl,} 1 \%$ Triton X-100, $5 \mathrm{mM}$ EDTA, $5 \mathrm{mM}$ DTT, $10 \mathrm{mM} \mathrm{NaF}, 1 \mathrm{mM}$ $\mathrm{Na}_{3} \mathrm{VO}_{4}, 1 \mathrm{mM}$ PMSF, and $50 \mu \mathrm{M}$ leupeptin. The lysate was precleared by centrifugation and then used for immunoprecipitation with anti-IRS-1 (Upstate Biotechnology, Lake Placid, NY, USA) or anti-IRS-2 (Upstate Biotechnology, Lake Placid, NY, USA) antibodies. The IRS-1 and the IRS-2 immunocomplexes were precipitated with protein-A sepharose. The immunoprecipitates were denatured by boiling in SDS- $\beta$-mercapthoethanol sample buffer and separated on 7\% SDS-PAGE. Proteins were transferred to Immobilon-P (Millipore) filters and analyzed by immunoblotting as described below.

For immunoprecipitation of IRS-1 in CBA and Shb islets, precultured islets in groups of 1000 were deprived from serum for $60 \mathrm{~min}$ and then stimulated with $10 \%$ FCS for 10 min. IRS-1 immunoprecipitation was then performed as described.

\section{Shb-SH2/PTB Domain Interactions with}

Focal Adhesion Kinase and IRS-2

The Shb SH2 domain (16) and PTB domain (17) GST fusion proteins were immobilized to Glutathione Sepharose (Amersham Pharmacia Biotech) beads and mixed on ice with lysates of control or insulin-stimulated RIN-Neo cells in the absence or presence of $20 \mathrm{mM}$ phosphotyrosine. After $30 \mathrm{~min}$, the beads were washed three times with PBS plus $1 \%$ Triton X-100 before SDS-PAGE and Western blotting, using $4 \mathrm{G} 10$ phosphotyrosine and focal adhesion kinase (FAK) antibodies.

\section{PI3K Assay}

RIN-Shb and RIN-Neo cells $\left(10 \times 10^{6}\right)$ were maintained in RPMI 1640 without serum for $60 \mathrm{~min}$. To some groups of cells insulin $(10 \mu \mathrm{g} / \mathrm{ml})$, FCS $(10 \%)$, or IGF-1 $(100 \mathrm{ng} / \mathrm{ml})$ was added. After 10 min, cells were washed in cold PBS and lysed 
in $100 \mathrm{mM}$ Tris, $\mathrm{pH} 8.0,150 \mathrm{mM} \mathrm{NaCl}, 1 \%$ Triton $\mathrm{X}-100,5 \mathrm{mM}$ EDTA, $5 \mathrm{mM}$ DTT, $10 \mathrm{mM} \mathrm{NaF}$, $1 \mathrm{mM} \mathrm{Na} \mathrm{VO}_{4}, \mathrm{l} \mathrm{mM}$ PMSF, and $50 \mu \mathrm{M}$ leupeptin. The lysate was precleared by centrifugation and then used for immunoprecipitation with an anti-phosphotyrosine antibody (PY20). The immunocomplexes were bound to Protein A sepharose (Amersham Pharmacia Biotech) and then washed two times with PBS containing 1\% NP-40 and $1 \mathrm{mM}$ DTT followed by two washes with 100 mM Tris, pH 7.6, $500 \mathrm{mM} \mathrm{LiCl,} \mathrm{and} 1 \mathrm{mM}$ DTT. The immunocomplexes were then washed once with $10 \mathrm{mM}$ Tris, pH 7.6, $100 \mathrm{mM} \mathrm{NaCl,} 1 \mathrm{mM}$ DTT, followed by incubation for $10 \mathrm{~min}$ in $10 \mathrm{mM}$ Tris, pH 7.6, $100 \mathrm{mM} \mathrm{NaCl}, 1 \mathrm{mM}$ DTT, and $0.25 \mathrm{mg} / \mathrm{ml}$ phosphatidylinositol. The reaction was initiated by addition of $10 \mathrm{mM} \mathrm{MgCl}_{2}, 20 \mathrm{mM}$ HEPES, pH 7.6, $50 \mu \mathrm{M}$ ATP (final concentrations in $50 \mu \mathrm{l}$ ) containing $5 \mu \mathrm{Ci}$ of $\gamma-\left[{ }^{32} \mathrm{P}\right]-\mathrm{ATP}$. After $10 \mathrm{~min}$, the reaction was terminated by the addition of $15 \mu \mathrm{l} 4 \mathrm{M} \mathrm{HCl}$ and $130 \mu \mathrm{l}$ chloroform/methanol $(1: 1, v / v)$. Phospholipids were extracted as previously described (25) and separated on silica TLC plates (Merck, Darmstadt, Germany) using $\mathrm{CHCl}_{3}: \mathrm{CH}_{3} \mathrm{OH}: \mathrm{NH}_{4} \mathrm{OH}: \mathrm{H}_{2} \mathrm{O}$ (600:470:20:113, v/v) as solvent.

\section{Immunoblot Analysis of Akt, ERK, and PTEN}

Shb and CBA islets (groups of 100), RIN-Shb, or RIN-Neo cells and GFP-RIN or GFP + Shb-RIN cells $\left(2-3 \times 10^{5}\right)$ were maintained in RPMI 1640 without serum for $60 \mathrm{~min}$. Some groups of cells or islets were then stimulated with insulin $(100 \mathrm{ng} / \mathrm{ml})$, FCS $(10 \%)$, or IGF-1 $(100 \mathrm{ng} / \mathrm{ml})$. After $10 \mathrm{~min}$, cells were washed in cold PBS and directly lysed in SDS- $\beta$-mercaptoethanol sample buffer containing 1 mM PMSF. Samples were then run on $7.5-12 \%$ SDS-PAGE and electroblotted to Immobilon-P filters. The filters were then incubated with rabbit polyclonal antibodies specific for phospho-Akt (Ser473), Akt, phospho-ERK, or ERK (New England Biolabs, Beverly, MA, USA) diluted 1:1000 in Tris-buffered saline supplemented with $2.5 \%$ bovine serum albumin (BSA). The filters were also analyzed for PTEN using mouse monoclonal anti-PTEN antibody (A2B1) (Santa Cruz Biotechnology, Santa Cruz, CA, USA). Horseradish peroxidase linked goat anti-rabbit or -mouse immunoglobulin (Ig) was used as a second layer. The immunodetection was performed as described for the ECL immunoblotting detection system (Amersham Pharmacia Biotech). The intensities of the bands were quantified by densitometric scanning using Kodak Digital Science ID software (Eastman Kodak, Rochester, NY, USA).

\section{PTEN Phosphorylation}

GFP-RIN and GFP + Shb-RIN cells $\left(2-3 \times 10^{5}\right)$ were washed in buffer A (30 mM HEPES, $110 \mathrm{mM}$ $\mathrm{NaCl}, 10 \mathrm{mM} \mathrm{KCl}, 1 \mathrm{mM} \mathrm{MgCl}_{2}, 10 \mathrm{mM}$ glucose,
$1.53 \mathrm{mM} \mathrm{Ca}^{2+}$, and $2 \mathrm{mg} / \mathrm{ml} \mathrm{BSA)} \mathrm{(26).} \mathrm{The} \mathrm{cells}$ were then labeled for $60 \mathrm{~min}$ in buffer A containing ${ }^{32} \mathrm{PO}_{4}(250 \mu \mathrm{Ci} / \mathrm{ml})$. Some groups of cells were then stimulated for $10 \mathrm{~min}$ with $10 \mu \mathrm{g} / \mathrm{ml}$ of insulin, followed by three washes with cold PBS. Cells were then washed, lysed, precleared, and used for immunoprecipitation of PTEN using the mouse monoclonal anti-PTEN antibody (A2B1) (Santa Cruz Biotechnology). To precipitate immunocomplexes, protein-G sepharose (Amersham Pharmacia Biotech) was used. The immunoprecipitates were run on $9 \%$ SDS-PAGE and transferred to Immobilon-P filters. The filters were exposed to x-ray film and the 55 $\mathrm{kDa}{ }^{32} \mathrm{P}$-band was verified to be phospho-PTEN by immunoblot analysis. The densitometric scannings of the ${ }^{32} \mathrm{P}$-autoradiography films were expressed per total ${ }^{32} \mathrm{P}$-protein incorporation.

\section{Results}

Effects of Shb Overexpression on RINm5F Cell Proliferation and Apoptosis

We previously observed that neonatal islets from Shb-transgenic mice display an increased $\beta$-cell mass, indicating enhanced $\beta$-cell proliferation (21). Because the supply of islet tissue is insufficient for careful studies of IRS-1-mediated signaling events, we utilized the RINm5F cell line with stable or transient Shb overexpression as a model. The RINm5F cell clone RIN-Shb (21) and RINm5F cells, the latter of which were transiently transfected with the Shb-pcDNAl vector (GFP + ShbRIN), both overexpress Shb (Fig. 1A). Shb overexpression is known to be associated with enhanced degradation of the protein, an event that can be partially prevented by incubating cells with the calpain inhibitor acetyl-leu-leu-norleucinal (21). Indeed, multiple Shb-related fragments are presently visible in the groups with Shb overexpression (Fig. 1A).

We next determined proliferation and apoptosis rates in transiently transfected Shb-overexpressing cells maintained at $10 \%$ FCS for 4 days following the transfection procedure. Transient Shb overexpression (GFP + Shb-RIN cells) resulted in increased proliferation rates as compared to GFP-transfected cells (GFP-RIN cells) (Fig. 2A). In addition, there was a nonsignificant trend toward less apoptosis in GFP + Shb-RIN cells as compared with the GFP-RIN cells (Fig. 2B). The rates of apoptosis were higher on day 1 following the sorting procedure as compared to day 3 (Fig. 2B). It appears as if the sorting procedure promotes cell death to a certain extent, which subsides after the first 2 days.

\section{Effects of Shb Overexpression on IRS-1 and IRS-2 Phosphorylation}

IRS-mediated signaling events are known to be intimately involved in the control of proliferation 
A

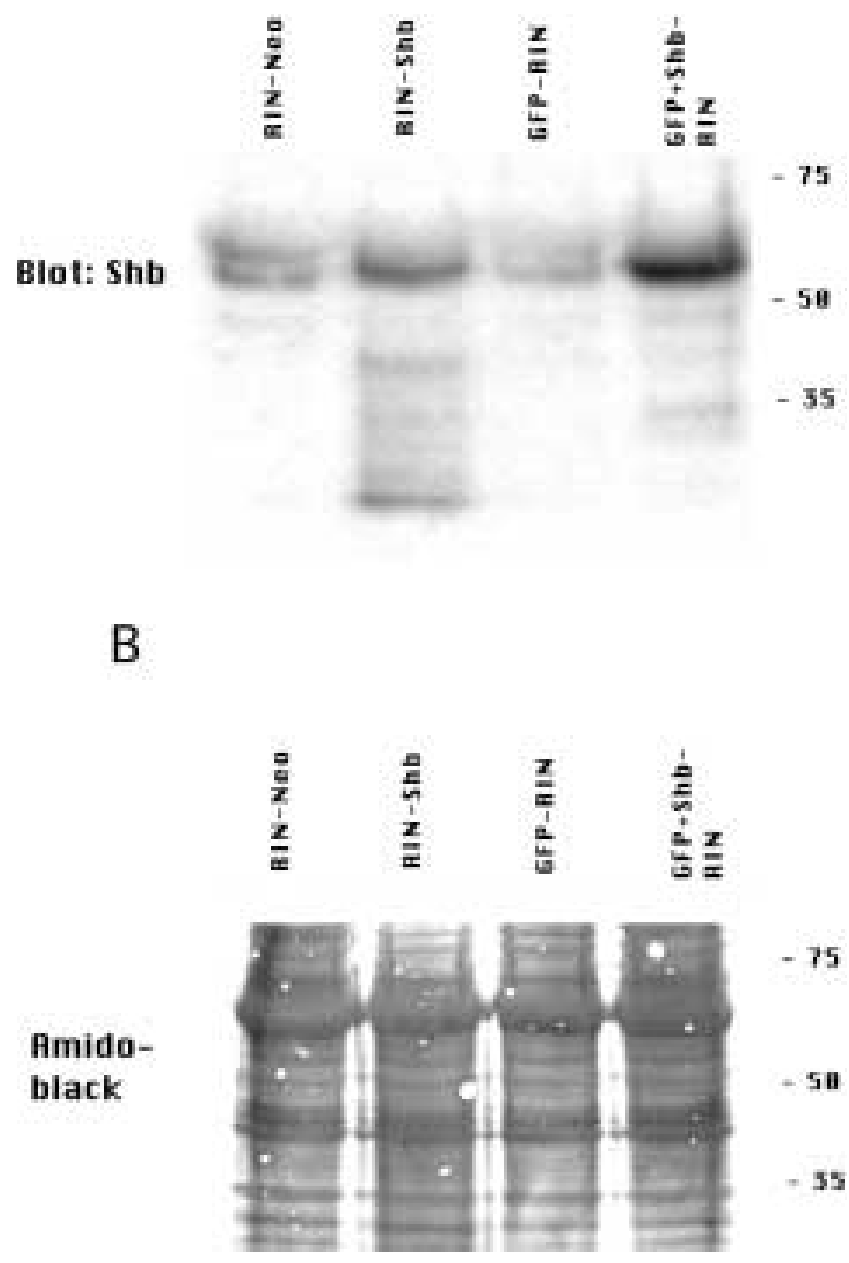

Fig. 1. Expression of Shb in RIN-Neo, Rin-Shb, GFP-RIN, and GFP + Shb-RIN cells. Stable clones selected for neomycin (RIN-Neo) or neomycin + Shb (RIN-Shb) expression, and RINm5F cells transiently expressing GFP-RIN or GFP + Shb-RIN were analyzed for Shb expression using an anti-Shb antibody (A). The GFP-transfected cells were sorted for green fluorescence 1 day after lipofection and analyzed by immunoblotting 1 day after the sorting procedure. The cells were treated for $20 \mathrm{~min}$ with $0.2 \mathrm{mM}$ acetyl-leu-leu-norleucinal prior to lysis in SDS-sample buffer. (B) Amido black staining (24) of the filter shown in (A) to visualize total protein loading and efficiency of transfer.

and cell survival. We therefore analyzed the effect of Shb overexpression on IRS-1 phosphorylation. Serum augmented the degree of tyrosine phosphorylation of IRS-1 in control islets, whereas the basal tyrosine phosphorylation of IRS-1 was higher in the islets isolated from Shb-transgenic mice compared with control islets (Fig. 3A). In the Shb-transgenic islets, serum failed to enhance further the tyrosine phosphorylation of IRS-1. Also, insulin stimulated the phosphorylation IRS- 1 in control islets, whereas no effect was noted in the SHB-transgenic islets.
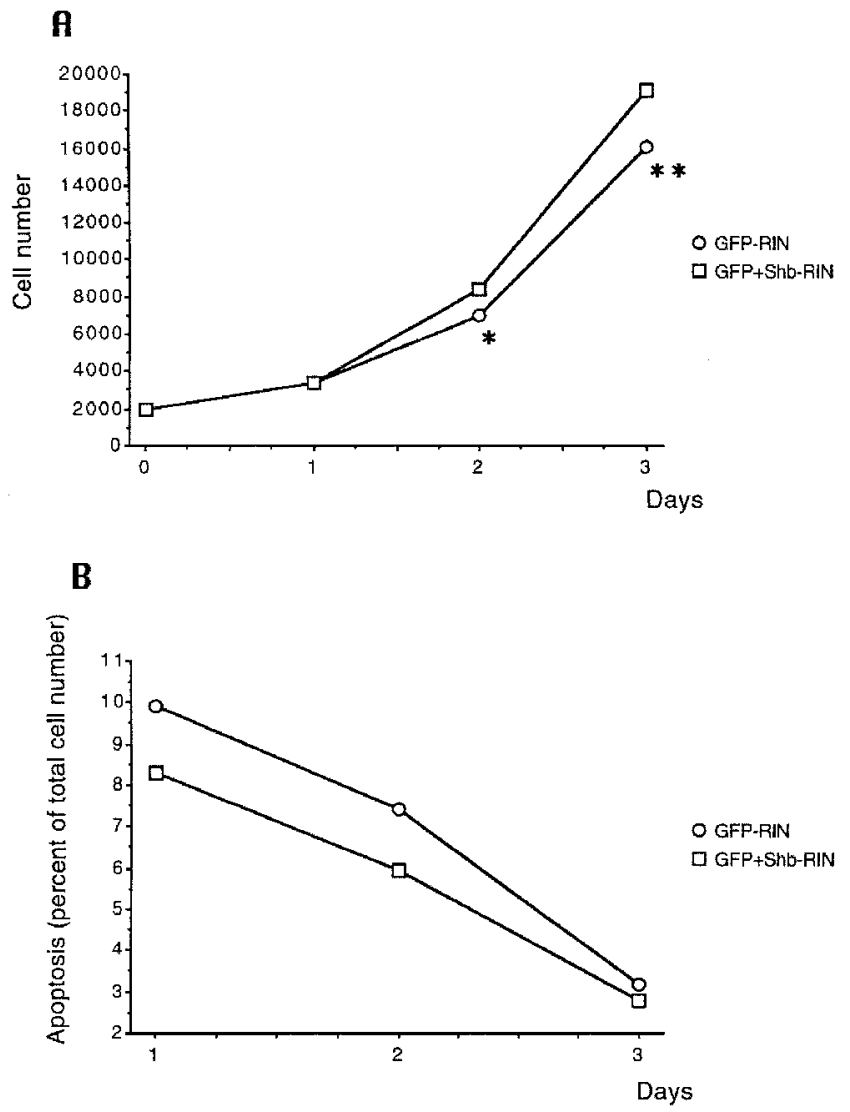

Fig. 2. Proliferation (A) and apoptosis (B) of GFP-RIN and GFP + Shb-RIN cells. 2000 GFP-RIN or GFP + Shb-RIN cells were plated in culture dishes on day 0 (day of cell sorting and one day after lipofection). On days 1, 2, and 3 cells were stained for $10 \mathrm{~min}$ with $10 \mu \mathrm{g} / \mathrm{ml}$ of PI and detached by trypsination. Cells were then counted (proliferation) and analyzed for $\mathrm{FL}_{3}$ fluorescence (apoptosis) by flow cytometry in a Becton-Dickinson FACSCalibur. Results are means for three independent observations. ${ }^{*} p<0.05$ and ${ }^{* *} p<0.01$ versus corresponding control using Student's paired $t$-test.

IRS-2 phosphorylation was increased under basal conditions in the SHB-transgenic islets, and insulin failed to cause a notable stimulation of IRS-2 phosphorylation in both groups of islets, presumably because of endogenous release of insulin from the islets, which was sufficient to saturate this response. Thus, Shb overexpression causes increased basal IRS-1 and IRS-2 phosphorylation in islets of Langerhans.

We next turned to the cell clone RIN-Shb, which continuously overexpresses Shb and provides an unlimited supply of cells to corroborate the findings of the islets of Langerhans. Control (RIN-Neo) or RINShb cells were maintained for $1 \mathrm{hr}$ in the absence of serum (0) or subsequently stimulated for $10 \mathrm{~min}$ with serum (S), insulin (Ins), or IGF-1. The cells were then collected, lysed, immunoprecipitated for IRS-1 or IRS-2, and subjected to immunoblotting for phosphotyrosine (4G10 antibody; Fig. 3B). Tyrosine phosphorylated IRS-1 was detected as a $180-\mathrm{kDa}$ 
B

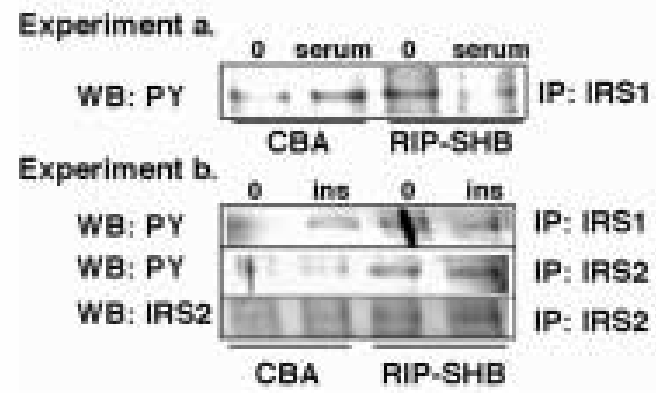

C

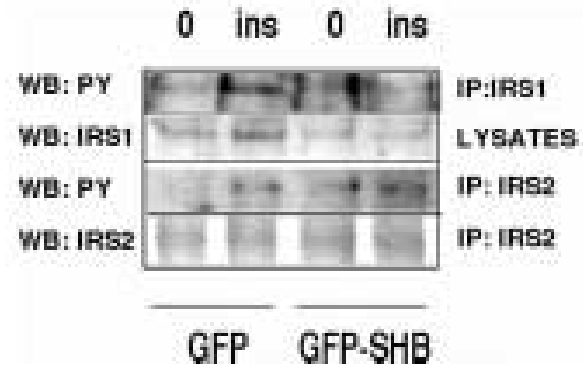

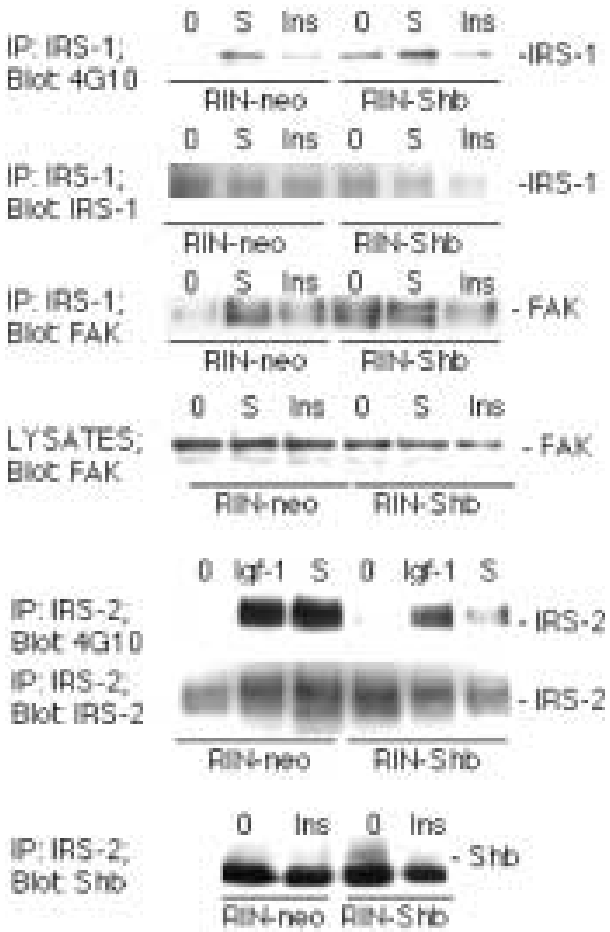

IP. IRS-1,

IP: IRS-1: Blok IAS-1

IP. IRS-1, BloC FAK

0 S ins $0 \quad S$ Ins

LYSATES:

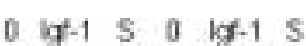
Blot 4010
IP: IAS-2,
Blot IRS-2

Fig. 3. IRS-1 and -2 tyrosine phosphorylation in islets isolated from Shb-transgenic mice (A), in RIN-neo and RIN-Shb cells (B), and in sorted GFP-RIN and GFP + Shb-RIN cells (C). (A) Islets (in groups of 1000) were isolated and cultured for 3-7 days before being incubated for $1 \mathrm{hr}$ in the absence of serum. In two groups of islets, these were further stimulated for 10 min with $10 \%$ FCS or $10 \mu \mathrm{g} / \mathrm{ml}$ insulin as indicated. The islets were then washed, lyzed, and immunoprecipitated with IRS-1 or IRS-2 antibody prior to immunoblot analysis for phosphotyrosine (4G10) or IRS-2. (B) Control (RIN-Neo) or Shb (RIN-Shb) cells (10 ${ }^{7}$ ) were maintained in the absence of serum for $1 \mathrm{hr}$ or additionally stimulated with $10 \%$ FCS (S), $10 \mu \mathrm{g} / \mathrm{ml}$ insulin (Ins), or $100 \mathrm{ng} / \mathrm{ml}$ IGF- 1 (Igf-1) for $10 \mathrm{~min}$, before being washed in PBS, scraped, and lysed for immunoprecipitation using IRS-1 or IRS-2 antibodies as indicated. Aliquots of the lysates were taken prior to immunoprecipitation and analyzed. The immunoprecipitations were subjected to immunoblot analysis and probed for phosphotyrosine (4G10), IRS-1, IRS-2, FAK, and Shb immunoreactivity. (C) GFP-RIN or GFP + Shb-RIN cells $\left(1-2 \times 10^{5}\right)$ were plated in culture dishes on day 0 (day of cell sorting and 1 day after lipofection). On day 1 , cells were deprived of serum for $60 \mathrm{~min}$, followed by addition of $100 \mathrm{ng} / \mathrm{ml}$ insulin for $10 \mathrm{~min}$ to some groups. The cells were then washed in PBS and lysed for immunoprecipitation using IRS-1 antibodies as indicated. The immunoprecipitates were subjected to immunoblot analysis and probed for phosphotyrosine (4G10).

band in the immunoprecipitates of the RIN-Neo and RIN-Shb cells. Shb overexpression enhanced the phosphorylation of IRS- 1 in the absence of stimulation (Figs. 3B and 4A). Whereas serum, insulin, or IGF- 1 all stimulated the tyrosine phosphorylation of IRS- 1 in the control cells, these additions failed to further increase IRS-1 phosphorylation in the RINShb cells (Figs. 3B, 4A).

To exclude the possibility that the effects of Shb were related to clonal selection, we also analyzed IRS-1 phosphorylation in transiently transfected RINm5F cells. Lipofection of RINm5F cells gives in our hands a transfection efficiency of only $4-10 \%$. However, by cotransfecting cells with both ShbpcDNAl and GFP vectors, Shb-expressing cells can be sorted by FACS and enriched to $>75 \%$ purity.
Unfortunately, a typical yield is only $1-2 \times 10^{5}$ sorted GFP-positive cells per culture dish. Thus, the number of cells available for analysis is limited, which results in a low signal/noise ratio when analyzing IRS-1 phosphorylation in these cells. Nevertheless, we observe that in sorted GFP-transfected cells ( $>75 \%$ transfected), addition of insulin stimulated IRS-1 phosphorylation (Fig. 3C). Furthermore, the phosphorylation of IRS-1 in the Shb cells is stronger at basal conditions and is not stimulated further by addition of insulin (Fig. 3C). Thus, transgenic Shb islets, transiently transfected RIN cells, and RIN-Shb cells all display a similar increase in basal IRS-1 phosphorylation.

IRS-2 phosphorylation in response to serum, insulin, or IGF-1 stimulation was reduced or 
A

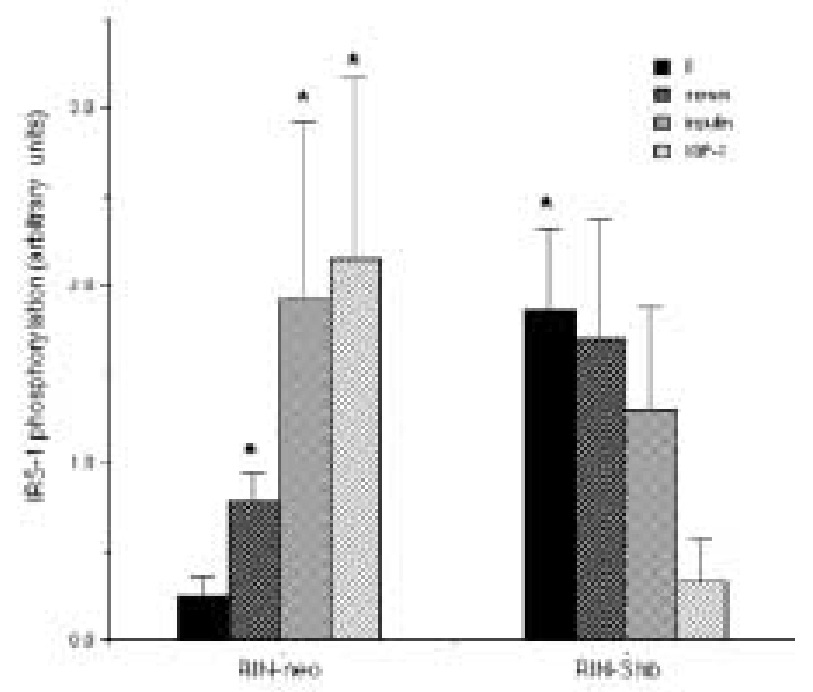

B

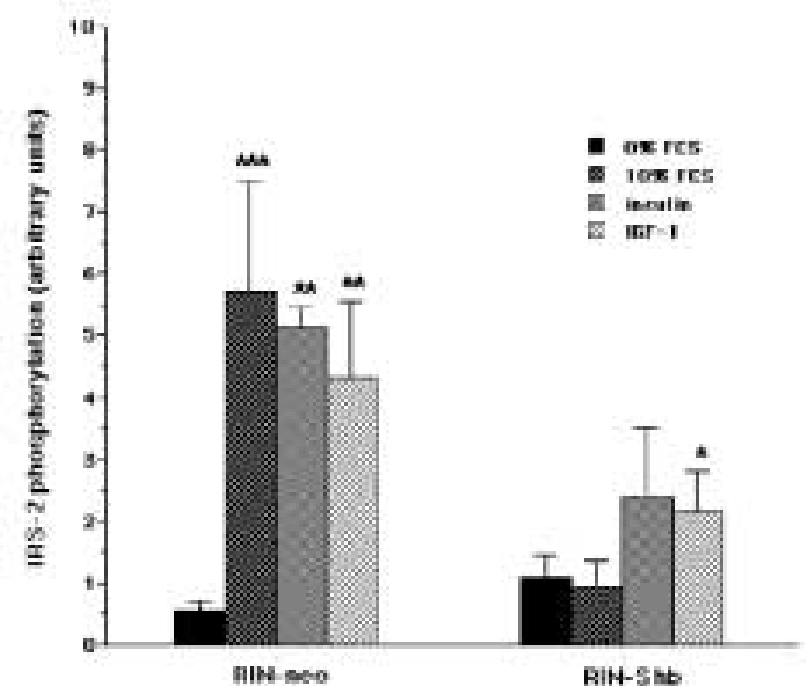

Fig. 4. Tyrosine phosphorylation of IRS-1 (A) and IRS-2 (B) in response to serum, insulin, and IGF-1 in RIN-Neo and RIN-Shb cells. The results in Figure 3B were subjected to densitometric analysis and means \pm SEM for 4-6 observations are given. The data were tested for statistical significance using one-way ANOVA on ranks $(p<0.05)$. Individual comparisons were performed using a paired Student's $t$-test comparing with corresponding untreated control.

completely abolished in the RIN-Shb cells concomitant with an increased basal level of IRS-2 phosphorylation (Figs. 3B, 3C, and 4B). The FAK was found to co-immunoprecipitate with IRS-1 in a manner that correlated with the degree of tyrosine phosphorylation of this substrate; the highest amounts of FAK were detected in the insulin-stimulated RINNeo cells and nonstimulated RIN-Shb cells (Fig. 3B). Shb itself co-immunoprecipitated with IRS-2 in nonstimulated RIN-Shb cells (Fig. 3B). The data suggest alterations in the tyrosine phosphorylation

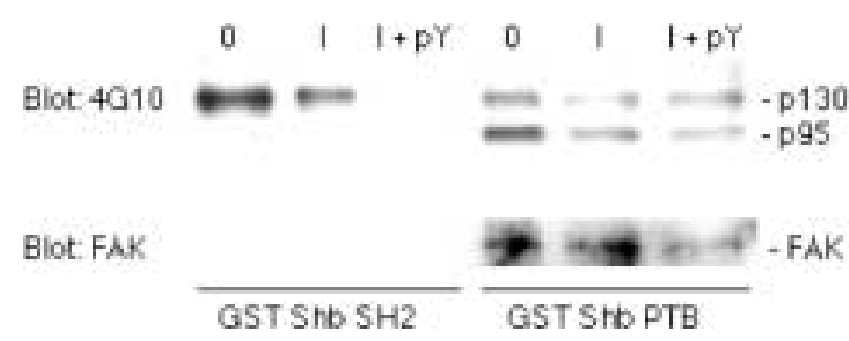

Fig. 5. Interactions of RINm5F cell proteins with Shb GST fusion proteins. Immobilized GST Shb SH2 and GST Shb PTB domain fusion proteins (10 $\mathrm{g}$ each) were mixed with cell lysates of nonstimulated or insulin-stimulated RINm5F cells. In one group, $20 \mathrm{mM}$ phosphotyrosine was included in the incubation mixture. After $30 \mathrm{~min}$, the beads were washed and subjected to immunoblot analysis for phosphotyrosine (4G10) and FAK.

and complex formation of IRS- 1 and IRS- 2 as a consequence of Shb overexpression.

\section{Interactions Between Shb and FAK}

The presence of Shb in the IRS-2 immunoprecipitates and FAK in the IRS-1 immunoprecipitates could relate to interactions between Shb and these molecules. To address this further, fusion proteins comprising the $\mathrm{SH} 2$ domain or PTB domain of Shb were used in pull-down experiments probing for phosphotyrosine, FAK, and IRS-2 by immunoblotting (Fig. 5). The Shb SH2 domain interacted with a 130-kDa phosphotyrosyl protein regardless of whether the cells were stimulated with insulin or not. Free phosphotyrosine effectively inhibited this interaction. Likewise, the PTB domain fusion protein bound two phosphotyrosyl proteins of 130 and $95 \mathrm{kDa}$. However, free phosphotyrosine could not displace these associations. When probing the blot using an anti-FAK antibody, two bands of 125 and $95 \mathrm{kDa}$ were noted using the PTB domain fusion protein. The $125-\mathrm{kDa}$ band corresponds to full-sized FAK, and was found to associate less well with the PTB domain fusion protein in the presence of free phosphotyosine (Fig. 5). This suggests a phosphotyrosinedependent association between Shb's PTB domain and FAK. This finding was recently corroborated in endothelial cells, in which the Shb PTB domain associates in a phosphotyrosine-dependent manner with FAK (27).

IRS-2 could not be detected in these fusion protein pull-down experiments (results not shown), suggesting either cooperativity between different domains of Shb for such an association, or the requirement of additional signaling molecules.

\section{Effect of Shb Overexpression on PI3K Activity}

Because PI3K is a downstream effector of IRS-1 and IRS-2 in their signaling pathways, we decided to investigate the effect of Shb overexpression on 


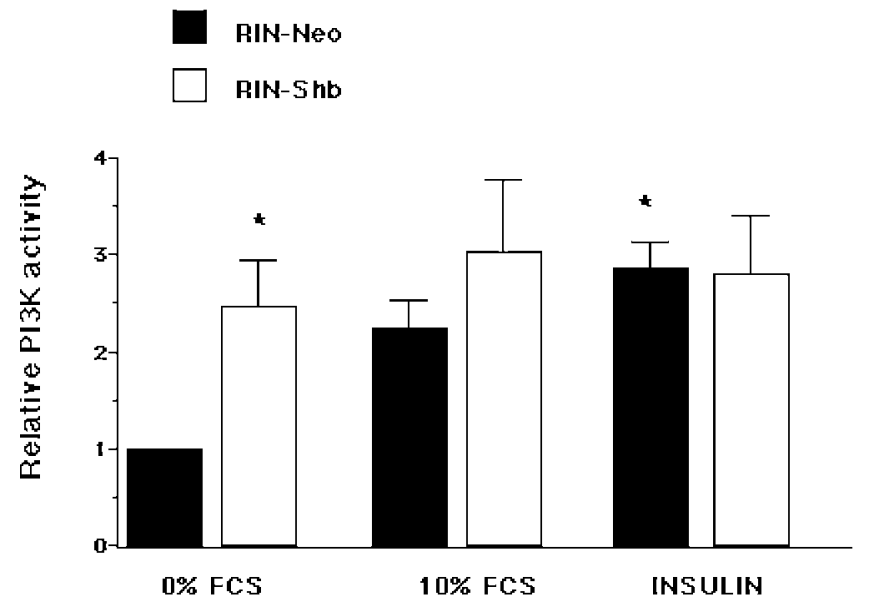

Fig. 6. Effects of FCS and insulin on PI3K activity in RINNeo and RIN-Shb cells. RIN-Neo and RIN-Shb cells were serum deprived for 60 min followed by stimulation with $10 \%$ FCS or $10 \mu \mathrm{g} / \mathrm{ml}$ insulin. Phosphotyrosine proteins were immunoprecipitated from homogenates and analyzed for PI3K activity. PI3K activity was related to the total homogenate protein content and expressed as fold-increase as compared with serum-deprived RIN-Neo cells. Results are means \pm SEM for three to eight observations. The data were analyzed for statistical significance using a one-way ANOVA on repeated measures $(p<0.05)$. Individual comparisons were performed using a Dunnet's test when comparing with the corresponding untreated controls.

the activity of this enzyme. RIN-Neo and RIN-Shb cells were used because we could not obtain sufficient quantities of islets or transiently transfected and sorted Shb-expressing cells. In the control RIN-Neo cells, serum and insulin increased the PI3K activity that was immunoprecipitated with the phosphotyrosine antibody PY20 (Fig. 6). The RIN-Shb cells displayed a significantly elevated basal PI3K activity and were not able to respond further to serum or insulin with increased activity. The data on PI3K activity reflects the combined IRS-1 and IRS-2 phosphorylation data (Figs. 4B and 4C) in that Shb overexpression increases basal activity and deregulates the response to stimulation with serum or insulin.

\section{Akt and ERK Phosphorylation}

Activation of Akt by serine phosphorylation requires increased PtdIns- $\mathrm{P}_{2 / 3}$ levels and leads to antiapoptotic signaling. Stimulation of sorted GFP-RIN cells with insulin resulted in increased Akt phosphorylation (Fig. 7). Sorted RINm5F cells transfected with GFP + Shb-cDNA, however, displayed a decreased insulin-induced Akt phosphorylation response, mainly due to increased basal phosphorylation (Fig. 7). These findings indicate that an enhanced PI3K-activity in response to Shb overexpression promotes Akt phosphorylation.

ERK phosphorylation was also increased in response to insulin in the control GFP-RIN cells (Fig. 7). The sorted SHB-RIN cells, however, failed
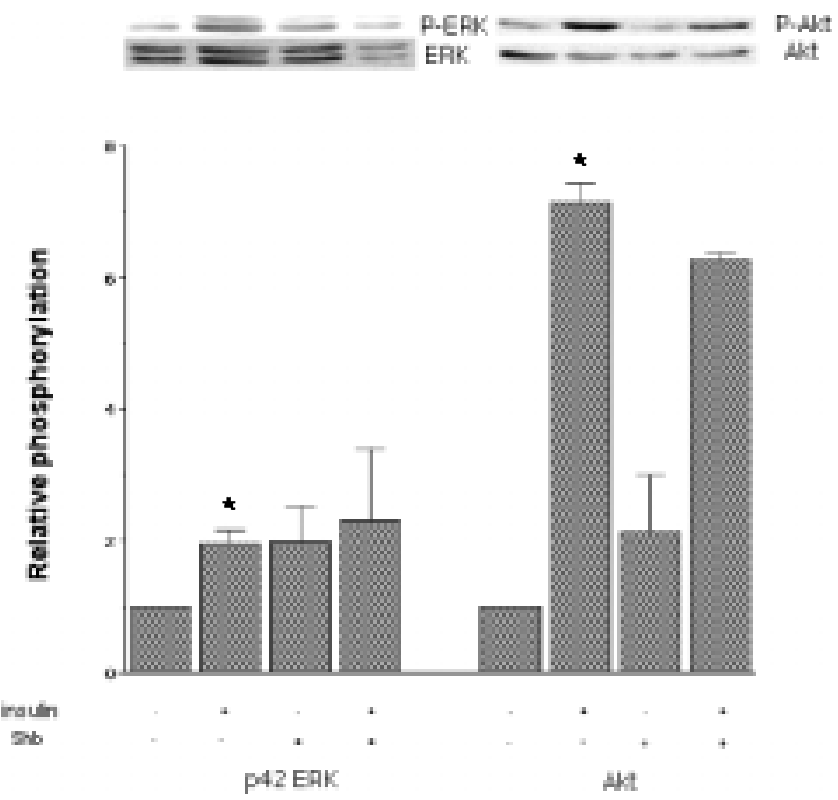

Fig. 7. Effects of insulin on ERK and Akt phosphorylation in GFP + Shb-RIN cells. Sorted or nonsorted GFP-RIN or GFP + Shb-RIN cells $\left(2 \times 10^{5}\right)$ were serum deprived 2 days after transfection for $60 \mathrm{~min}$ followed by stimulation with $100 \mathrm{ng} / \mathrm{ml}$ insulin for $10 \mathrm{~min}$. Cells were lysed in SDS- $\beta$-mercapthoethanol buffer, run on $9 \%$ SDS-PAGE gels, and immunoblotted for phospho-ERK, total ERK, phospho-Akt, and total Akt expression. Filters were stripped in SDS- $\beta$-mercaptoethanol solution between blotting with the two different antibodies. Results from three separate experiments are expressed as means \pm SEM. ${ }^{*} p<0.05$ using Students paired $t$-test.

to increase their ERK-phosphorylation in response to insulin, mainly due to increased basal ERK phosphorylation (Fig. 7). Thus, Shb overexpression causes perturbed signaling through both the PI3K/Akt and ERK pathways.

\section{PTEN Levels and PTEN Phosphorylation}

We also analyzed the cellular contents of the phosphoinositide-dephosphorylating enzyme PTEN, which antagonizes the activity of PI3K. Immunoblot analysis revealed that levels of PTEN, expressed as optical density per amount of protein applied to each lane, in cell extracts from RIN-Shb cells and Shb islets, were not significantly different from those of corresponding control cells: $2.1 \pm 0.34$ (RIN-Neo, $n=4) 2.9 \pm 0.61$ (RIN-Shb, $n=4, p>$ 0.05 ), $1.1 \pm 0.17$ (Neo-islets, $n=9$ ) and $1.4 \pm 0.16$ (Shb islets, $n=9, p>0.05$ ). These findings do not support the notion that the expression of the PTEN gene is significantly altered in response to Shb overexpression.

Interestingly, both sorted GFP-RIN and GFP + Shb-RIN cells responded to insulin with a modest increase in PTEN phosphorylation (Fig. 8). This increase was slightly less pronounced in GFP-RIN cells and did not reach statistical significance, as opposed to the effect of insulin in GFP + Shb-RIN cells (Fig. 8). 
A
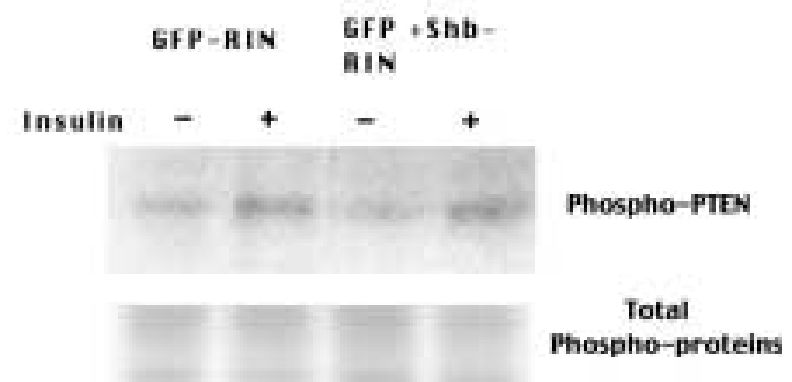

B

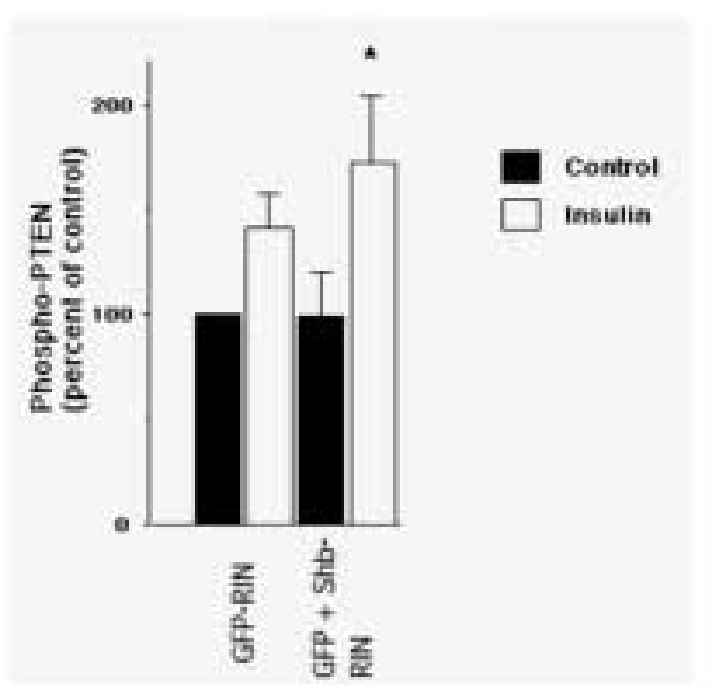

Fig. 8. Effects of insulin on PTEN phosphorylation in GFP-RIN and GFP + Shb-RIN cells. GFP-RIN or GFP + ShbRIN cells $\left(2 \times 10^{5}\right)$ were labeled for 60 min with ${ }^{32} \mathrm{P}$-phosphate and then stimulated for 10 min with insulin $(10 \mu \mathrm{g} / \mathrm{ml})$. PTEN was immunoprecipitated, electrophoresed on a $9 \%$ SDS-PAGE, and transferred to an Immobilon filter, which was exposed to $\mathrm{x}$-ray film. Nonprecipitated lysates were run in parallel. The autoradiographic bands shown in (A) were quantified and expressed per total protein ${ }^{32} \mathrm{P}$-incorporation (B). Results are means \pm SEM for three to four observations. Comparisons were made using one-way ANOVA for repeated measurements $(p<0.05)$. Individual differences were obtained using Student Newman Keuls test. ${ }^{*} p<0.05$ versus corresponding control cells.

\section{Discussion}

We presently describe major perturbations in IRS-1 and IRS- 2 signaling in insulin producing $\beta$-cells as a consequence of overexpression of the $\mathrm{SH} 2$ domain adapter protein Shb. Shb consists of a C-terminal SH2 domain, a central PTB domain, several potential tyrosine phosphorylation sites, and N-terminal proline-rich motifs. Thus, Shb participates in multiprotein complex formation as a consequence of tyrosine kinase signaling. The alterations presently observed are increased basal phosphorylation of IRS-1, both in response to short- and long-term Shb overexpression, and reduced serum- or insulin-stimulated phosphorylation of IRS-2 in cells with longterm Shb overexpression. In addition, increased association of FAK with IRS-1 was observed in the nonstimulated Shb cells. The alterations of IRS-1 and IRS-2 phosphorylation are presumably a consequence of Shb-induced complex formation. One such interaction presently observed is that between FAK and the Shb PTB domain. In addition, Shb associated with IRS-2 and this interaction could not be detected by fusion protein pull-down experiments. Thus, a third component may be required for this association to occur or full-sized Shb may be necessary for its binding to IRS-2. Nevertheless, the data strongly suggest that Shb via its domain interactions promotes multi-signaling protein complex formation, and that this has a major impact on the IRSsignaling pathways.

FAK is known to play a key part in integrin signaling pathways, which are engaged in the response to extracellular matrix interactions. The presently observed Shb-induced recruitment of FAK to IRS-1 supports and extends previous observations in glioblastoma cells and human embryo kidney cells demonstrating that FAK binds to IRS- 1 and that integrin-mediated FAK-activation and growth factor receptor activation converge at the IRS-1 protein $(27,28)$. Both FAK and IRS-1 have been reported to interact with PI3K (29) and all three proteins are known to have anti-apoptotic properties (30-32). In addition, integrin engagement is known to potentiate PDGF and insulin induced DNA synthesis (33). Taken together, these circumstances support the notion that Shb overexpression amplifies integrin-induced cell growth and survival signals by promoting multisignaling protein complex formation. This could explain the presently observed increase in GFP + ShbRIN cell proliferation, the trend to a lower rate of apoptosis in the presence of $10 \%$ serum, as well as the increased $\beta$-cell mass of neonatal and young Shbtransgenic mice (21). Indeed, the islets of these mice have an increased DNA content, insulin content, and insulin release, and this is paralleled by an improved glucose tolerance on an intravenous glucose tolerance test (21). Thus, during fetal development, Shb might potentiate the signals originating from $\beta$-cell interaction with the extracellular matrix and circulating growth factors leading to PI3K and Akt kinase activation. Indeed, a recent study has shown that Shbtransgenic mice display an increased $\beta$-cell replication in response to partial pancreatectomy (34). However, the insulin content expressed per DNA was not increased in Shb islets (21), which might indicate that the expression of the insulin gene is not under the direct control of the Shb-induced FAK/IRS-1 signaling pathway, only the proliferation.

Overexpression of Shb leads not only to an increased $\beta$-cell mass during the fetal and neonatal stages, but also to a higher sensitivity at adulthood 
to apoptotic signals such as those of serum deprivation, cytokines, and inhibition of poly(ADP-ribose) synthase (21). This is somewhat surprising because the increased PI3K activity observed in serumdeprived RIN-Shb cells argue in favor of an increased proliferation and a decreased sensitivity to apoptotic signals. It could therefore be speculated that following the initial proliferation (fetal) phase, driven by an increased IRS phosphorylation and enhanced PI3K-activity, a putative Shb-induced pro-apoptotic signal, predominates over the increased IRS signaling leading to cessation of stimulated proliferation and an enhanced susceptibility to apoptosis.

Our data suggest that Shb overexpression affects neither expression nor phosphorylation of PTEN in insulin-producing cells. However, we do observe an increased phosphorylation of PTEN in response to insulin. It is not clear how insulin stimulates PTEN phosphorylation in RINm5F cells, nor is the physiologic relevance of this event known. In a recent study, it was demonstrated that the 70-aa long Cterminal domain of PTEN (also called the tail) is serine/threonine phosphorylated in vivo, and that this event results in decreased activity and enhanced stability of the protein (35). More specifically, it appears that the tail restricts the activity of the catalytic phosphatase domain and that this effect is less pronounced if the phosphates at the tail are not present (35). Thus, it could be speculated that insulin, by promoting PTEN phosphorylation, inhibits dephosphorylation of phosphoinositides leading to a more pronounced response. On the other hand, an additional study has identified the protein kinase that phosphorylates the tail of PTEN to be casein kinase II (36). In addition, phosphorylation of PTEN in COS-7 cells by this kinase was not regulated in response to insulin (36). Therefore, further studies are clearly warranted to characterize the insulininduced PTEN-phosphorylation event in insulinproducing cells and its physiologic relevance.

In summary, we have elucidated interactions between Shb and the IRS-1/2/PI3K/Akt/PTEN/ERK pathways and found that these interactions are paralleled by enhanced proliferation. The understanding of the regulation of this pathway will be of importance for the clarification of $\beta$-cell apoptosis and proliferation in diabetes mellitus. It is also possible that these signaling steps are involved in the physiologic autocrine regulation by insulin of $\beta$-cell function, and in the putative pathologic insulin resistance of $\beta$-cells $(37,6)$. It has been proposed that prolonged periods of peripheral insulin resistance, hyperinsulinemia and cytokine exposure lead to $\beta$-cell insulin resistance and an impaired insulin secretion (37-39).

\section{Acknowledgments}

The excellent technical assistance of Ing-Marie Mörsare and Ing-Britt Hallgren is gratefully acknowledged. This work was supported by grants from the
Swedish Medical Research Council (12X-109, 12X11564, 31X-10822, 72P-12995), the Swedish Diabetes Association, the Nordic Insulin Fund, the Juvenile Diabetes Foundation International, the Wallenberg Foundation, and the Family Ernfors Fund.

\section{References}

1. Weir GC, Bonner-Weir S, Leahy JL. (1990) Islet mass and function in diabetes and transplantation. Diabetes 39: 401-405.

2. Brüning JC, Winnay J, Bonner-Weir S, Taylor SI, Accili D, Kahn CR. (1997) Development of a novel polygenic model of NIDDM in mice heterozygous for IR and IRS-1 null alleles. Cell 88: 561-572.

3. Withers DJ, Gutierrez JS, Towery H, et al. (1998) Disruption of IRS-2 causes type 2 diabetes in mice Nature 391: 900-904.

4. Withers DJ, Burks DJ, Towery HH, Altamuro SL, Flint CL, White MF. (1999) Irs-2 coordinates Igf-1 receptor-mediated beta-cell development and peripheral insulin signalling. Nature Gen. 23: 32-40.

5. Leibiger IB, Leibiger B Moede T, Berggren PO. (1998) Exocytosis of insulin promotes insulin gene transcription via the insulin receptor/PI-3 kinase/p70 s6 kinase and CaM kinase pathways. Mol. Cell 1: 933-938.

6. Kulkarni RN, Brüning JC, Winnay JN, Postic C, Magnuson MA, Kahn CR. (1999) Tissue-specific knockout of the insulin receptor in pancreatic beta cells creates an insulin secretory defect similar to that in type 2 diabetes. Cell 96: 329-339.

7. Whitman M, Downes CP, Keeler M, Keller T, Cantley L. (1988) Type I phosphatidylinositol kinase makes a novel inositol phospholipid, phosphatidylinositol-3-phosphate. Nature 332: 644-646.

8. Franke TF, Kaplan DR, Cantley LC, Toker A. (1997) Direct regulation of the Akt proto-oncogene product by phosphatidylinositol-3,4-bisphosphate. Science 275: 665-668.

9. Stokoe D, Stephens LR, Copeland T, et al. (1997) Dual role of phosphatidylinositol-3,4,5-trisphosphate in the activation of protein kinase B. Science 277: 567-570.

10. Franke TF, Kaplan D, Cantley L. (1997) PI3K: downstream AKTion blocks apoptosis. Cell 88: 435-437.

11. Datta SR, Dudek H, Tao X, et al. (1997) Akt phosphorylation of BAD couples survival signals to the cell-intrinsic death machinery. Cell 91: 231-241.

12. Cardone MH, Roy N, Stennicke HR, et al. (1998) Regulation of cell death protease caspase-9 by phosphorylation. Science 282: 1318-1321.

13. Brunet A, Bonni A, Zigmond MJ, et al. (1999) Akt promotes cell survival by phosphorylating and inhibiting a Forkhead transcription factor. Cell 96: 857-868.

14. Maehama T, Dixon JE. (1998) The tumor suppressor, PTEN/MMACl, dephosphorylates the lipid second messenger, phosphatidylinositol 3,4,5-trisphosphate. J. Biol. Chem 273: 13375-13378.

15. Cantely LC, Neel BG. (1999) New insights into tumor suppression: PTEN suppresses tumor formation by restraining the phosphoinositide 3-kinase/AKT pathway. Proc. Natl. Acad. Sci. U. S. A. 96: 4240-4245.

16. Welsh M, Mares J, Karlsson T, Lavergne C, Bréant B, ClaessonWelsh L. (1994) Shb is a ubiquitously expressed Src homology 2 protein. Oncogene 9: 19-27.

17. Welsh M, Songyang Z, Frantz D, et al. (1998) Stimulation through the $T$ cell receptor leads to interactions between SHB and several signaling proteins Oncogene 16: 891-902.

18. Karlsson T, Songyang Z, Landgren E, et al. (1995) Molecular interactions of the Src homology 2 domain protein Shb with phosphotyrosine residues, tyrosine kinase receptors and Src homology 3 domain proteins Oncogene 10: 1475-1483.

19. Lindholm CK, Gylfe E, Zhang W, Samelson LE, Welsh M. (1999) Requirement of the Src homology 2 domain protein 
Shb for $\mathrm{T}$ cell receptor-dependent activation of the interleukin-2 gene nuclear factor for activation of $\mathrm{T}$ cells element in Jurkat T cells. J. Biol. Chem. 274: 28050-28057.

20. Karlsson T, Welsh M. (1996) Apoptosis of NIH3T3 cells overexpressing the Src homology 2 domain protein Shb. Oncogene 13: 955-961.

21. Welsh M, Christmansson L, Karlsson T, Sandler S, Welsh N. (1999) Transgenic mice expressing Shb adaptor protein under the control of rat insulin promoter exhibit altered viability of pancreatic islet cells. Mol. Med. 5: 169-180.

22. Andersson A. (1978) Isolated mouse pancreatic islets in culture: effects of serum and different culture media on the insulin production of the islets. Diabetologia 14: 397-404.

23. Saldeen J, Curiel DT, Eizirik DL, et al. (1996) Efficient gene transfer to dispersed human pancreatic islet cells in vitro using adenovirus-polylysine/DNA complexes or polycationic liposomes. Diabetes 45: 1197-1203.

24. Saldeen J, Lee JC, Welsh N. (2001) Role of p38 mitogenactivated protein kinase (p38 MAPK) in cytokine-induced rat islet cell apoptosis. Biochem. Pharmacol. 61:1561-1569.

25. Welsh N. (1996) Interleukin-1 beta-induced ceramide and diacylglycerol generation may lead to activation of the c-Jun NH2-terminal kinase and the transcription factor ATF2 in the insulin-producing cell line RINm5F J. Biol. Chem. 271: 8307-8312.

26. Traynor-Kaplan AE, Thompson BL, Harris AL, Taylor $\mathrm{P}$, Omann GM, Sklar LA. (1989) Transient increase in phosphatidylinositol 3,4-bisphosphate and phosphatidylinositol trisphosphate during activation of human neutrophils. J. Biol. Chem. 264: 15668-15673.

27. Homqvist K, Cross M, Riley D, Welsh M. (in press) The Shb adaptor protein causes Sic-dependent cell spreading and activation of focal adhesion kinase in murine brain endothelial cells. Cell. Signal.

28. Sonoda Y, Watanabe S, Matsumoto Y, Aizu-Yokota E, Kasahara T. (1999) FAK is the upstream signal protein of the phosphatidylinositol 3-kinase-Akt survival pathway in hydrogen peroxide-induced apoptosis of a human glioblastoma cell line. J. Biol. Chem. 274: 10566-10570.

29. Lebrun P, Mothe-Satney I, Delahaye L, Obberghen EV, Baron V. (1998) Insulin receptor substrate-1 as a signaling molecule for focal adhesion kinase pp125(FAK) and pp60(src). J. Biol. Chem. 273: 32244-32253.

30. Chen HC, Appeddu PA, Isoda H, Guan JL. (1996) Phosphorylation of tyrosine 397 in focal adhesion kinase is required for binding phosphatidylinositol 3-kinase. J Biol. Chem. 271: 26329-26334.

31. Hungersford JE, Compton MT, Matter ML, Hoffstrom BG, Otey CA. (1996) Inhibition of pp125FAK in cultured fibroblasts results in apoptosis. J. Cell. Biol. 135: 1383-1390.

32. Ueno $H$, Honda $H$, Nakamoto $T$, et al. (1997) The phosphatidylinositol 3' kinase pathway is required for the survival signal of leukocyte tyrosine kinase. Oncogene 14: 3067-3072.

33. Zhou LF, Xu SQ, Dews M, Baserga R. (1997) Co-operation of simian virus $40 \mathrm{~T}$ antigen and insulin receptor substrate- 1 in protection from apoptosis induced by interleukin-3 withdrawal. Oncogene 15: 961-970.

34. Schneller M, Vuori K, Ruoslahti E. (1997) Alphavbeta3 integrin associates with activated insulin and PDGFbeta receptors and potentiates the biological activity of PDGF. EMBO J. 16: $5600-5607$.

35. Anneren C. (2002) Dual role of the tyrosine kinase GTK and the adaptor protein SHB in beta-cell growth: enhanced betacell replication after $60 \%$ pancreatectomy and increased sensitivity to streptozotocin. J. Endocrinol. 172: 145-153.

36. Vazquez F, Ramaswamy S, Nakamura R, Sellers WR. (2000) Phosphorylation of the PTEN tail regulates protein stability and function. Mol. Cell. Biol. 20: 5010-5018.

37. Torres J, Pulido R. (2001) The tumor suppressor PTEN is phosphorylated by the protein kinase CK2 at its C terminus. Implications for PTEN stability to proteasome-mediated degradation. J. Biol. Chem. 276: 993-998.

38. Rothenberg PL, Willison LD, Simon J, Wolf BA. (1995) Glucose-induced insulin receptor tyrosine phosphorylation in insulin-secreting beta-cells. Diabetes 44: 802-809.

39. Kwon G, Xu G, Marshall CA, McDaniel ML. (1999) Tumor necrosis factor alpha-induced pancreatic beta-cell insulin resistance is mediated by nitric oxide and prevented by 15-deoxy-Delta 12,14-prostaglandin $\mathrm{J} 2$ and aminoguanidine. A role for peroxisome proliferator-activated receptor gamma activation and inos expression. J. Biol. Chem. 274: 18702-18708. 